\title{
Spinal Cord Stimulation: An Update
}

\author{
Steven Falowski, Amanda Celii, and Ashwini Sharan \\ Department of Neurosurgery, Jefferson Medical College, Philadelphia, Pennsylvania 19107
}

\begin{abstract}
Summary: Spinal cord stimulation has been used in the treatment of many chronic pain disorders since 1967. In this update, the indications for spinal cord stimulation are reviewed with attention to recent publications. A focused review of the literature on abdominal and visceral pain syndromes is also provided. Furthermore, the technology has evolved from the use of monopolar electrodes to complex electrode arrays. Similarly,
\end{abstract}

the power source has changed from a radio frequency-driven system to a rechargeable impulse generator. These topics are covered, along with a short discussion of implant technique. Finally, we include a review of complications of such therapy. SCS as a technology and therapy continues to evolve. Key Words: Spinal cord stimulation, dorsal column stimulation, chronic pain, neuropathic pain, pacemaker.

\section{INTRODUCTION}

Spinal cord stimulation (SCS) is an adjustable, nondestructive, neuromodulatory procedure that delivers therapeutic doses of electrical current to the spinal cord for the management of neuropathic pain. The most common indications include postlaminectomy syndrome, complex regional pain syndrome, ischemic limb pain, and angina. Scattered reports have also appeared regarding the treatment of intractable pain due to other causes including visceral and abdominal pain, cervical neuritis pain, spinal cord injury pain, postherpetic neuralgia, and neurogenic thoracic outlet syndrome. The procedures are most commonly performed by neurosurgeons or anesthesiologists specializing in pain management, ${ }^{1-3}$ but other specialties, such as rehabilitation medicine and orthopedic surgery, have also demonstrated interest in the procedure.

Enthusiasm for SCS began with the introduction of the gate-control theory for pain control by Melzack and $\mathrm{Wall}^{4}$ in 1965. They noted that stimulation of large myelinated fibers of peripheral nerves resulted in paresthesias and blocked the activity in small nociceptive projections. In 1967, Shealy inserted the first dorsal column stimulator in a human suffering from terminal metastatic cancer. ${ }^{5,6}$ Subsequently, electrodes have been implanted utilizing a variety of techniques: via a laminectomy in

Address correspondence and reprint requests to: Ashwini D. Sharan, M.D., Department of Neurosurgery, Jefferson Medical College, 909 Walnut Street, 2nd Floor, Philadelphia, PA 19107. E-mail: ashwini. sharan@jefferson.edu. the subarachnoid space ${ }^{6}$ or between the two layers of the dura or in the epidural space either dorsal or ventral to the spinal cord. ${ }^{7-9}$ Subsequently, less invasive percutaneous techniques were introduced. ${ }^{10}$

Great advances in technology have also driven the popularity of the field. The electrodes were initially all unipolar; the bipolar arrays developed subsequently. Furthermore, the contact combinations could only be hardwired, and could not be reprogrammed after the pulse generator was implanted. In the beginning, only radio frequency-driven (RF-driven) passive receivers were available. In the mid-1970s, the first implantable pulse generator powered by a lithium battery was introduced into the market. In 1980, the first percutaneous quadripolar electrode was produced, one that could be reprogrammed noninvasively through an external transmitter. ${ }^{11}$ Subsequently, implantable pulse generators (IPG) have been developed that can be both transcutaneously charged and programmed. This most recent advance is now leading a renewed interest in the utility of using special electrode arrays in the delivery of electrical stimulation to the spinal cord.

\section{MECHANISMS OF ACTION}

Although a large body of work has been published, the exact mechanisms of action of SCS remain unclear. The computer modeling studies of Holsheimer, Coburn, and Struijk ${ }^{12-17}$ have shed some light, at least theoretically, on the distribution of the electrical fields within the spinal structures. It is clear that stimulation on the dorsal 
aspect of the epidural space creates complex electrical fields that affect a large number of structures. We do not know whether activating afferents within the peripheral nerve, dorsal columns, or supralemniscal pathways share equivalent mechanisms of action. Furthermore, there may be antidromic action potentials passing caudally in the dorsal columns to activate spinal segmental mechanisms in the dorsal horns, as well as action potentials ascending in the dorsal columns activating cells in the brainstem, which in turn might drive descending inhibition. At the chemical level, animal studies suggest that the SCS triggers the release of serotonin, substance P, and GABA within the dorsal horn. ${ }^{18-20}$

\section{INDICATIONS}

SCS has been used for a variety of pain conditions and is particularly indicated for pain of neuropathic origin, including postlaminectomy syndrome, complex regional pain syndrome, phantom limb pain, spinal cord injury pain, and interstitial cystitis. The indications have been extended to include intractable pain due to abdominal or visceral pain and neurogenic thoracic outlet syndrome. SCS has been used successfully to treat severe pain due to ischemic disease of the lower extremities and, more recently, intractable angina pain. Experience suggests that, in selected patients, SCS can produce at least 50\% pain relief in $50-60 \%$ of the implanted patients. Notably, with proper follow-up care, these results can be maintained over several years.

\section{Complex regional pain syndrome}

The implementation of SCS in individuals with complex regional pain syndrome type I is more difficult than with any other patient group. The possibility of aggravating the original pain or causing a new pain or allodynia at the implanted hardware site is greater than with any other diagnostic category mentioned. The pain may spread to other body parts, and it is challenging to be able to cover all the affected areas with stimulation.

In 1989, Barolat et al. ${ }^{21}$ reported reduction of pain in 10 of 13 patients implanted. No patients in that series were made pain-free but all 10 reported a definitive difference when the stimulation was stopped. In 1997, Kumar et al. ${ }^{22}$ presented a median follow-up of 41 months on 12 patients with permanently implanted leads; 8 patients reported near complete resolution of their symptoms and the remaining 4 maintained good relief.

In another series, Kemler et al. ${ }^{23}$ reported 23 additional cases, with $78 \%$ of the patients reporting improvement. In 2000, Kemler et al. ${ }^{24}$ published work on a series of 54 patients who underwent randomization either to SCS with physical therapy or to physical therapy alone. In the SCS group, 67\% patients experienced significant pain relief, which persisted at 6 months. A 2.4-cm decrease and improvement in visual analog pain score (VAS) was noted in the SCS group, compared with 0.2-cm increase in the physical therapy group; however, no functional improvement was observed in either group. In 2006, in a letter to the editor of the New England Journal of Medicine, Kemler et al. ${ }^{25}$ recounted their 5-year follow-up on the patients with SCS. Their major conclusion was that the effects of SCS diminished over time for these patients; they did not specify what impact reprogramming or modern devices might have on the long-term effects of SCS therapy.

Oakley and Weiner ${ }^{26}$ reported a prospective study of 19 patients with complex regional pain syndrome implanted with spinal cord stimulation systems. Of the 10 patients for whom detailed long-term efficacy data were available, 3 reported full relief from their pain and 7 reported partial relief.

Including the Oakley and Weiner study, three prospective studies without matched controls have been reported (total of 50 subjects). ${ }^{26-28}$ Two of the studies reported success rates, with an $84 \%$ overall success rate. The study by Calvillo et al. ${ }^{27}$ reported a significant improvement in pain scores (VAS) and a $>50 \%$ reduction in narcotic use by $44 \%$ of subjects. In eight retrospective studies, the overall success rate was 84\% (192 patients). ${ }^{29}$

\section{Postlaminectomy syndrome}

Postlaminectomy syndrome (also called failed back surgery syndrome) is vaguely defined. The term has included pain localized to the center of the lower lumbar area, pain in the buttocks, persistent radicular pain, or diffuse lower extremity pain. Arachnoiditis, epidural fibrosis, radiculitis, microinstability, recurrent disk herniations, and infections have been implicated in the etiology of this syndrome. Most published series distinguish between back and leg pain, but the details of the pain syndromes are seldom defined. SCS is accepted in the treatment of leg pain, but its widespread use for relief of pain in the lower lumbar area still remains to be defined.

A great challenge in the treatment of postlaminectomy syndrome has been to obtain stimulation in the low back. Even with direct stimulation to the low back, the pattern of paresthesia is often replaced in time by an unpleasant segmental band of stimulation from the thoracic roots, which negates the benefits of the procedure. Previous pioneering work by Jay Law ${ }^{30,31}$ has shown that stimulation in the low back can be obtained only if one uses multiple arrays of closely spaced bipoles at T9-T10. North et al. ${ }^{32}$ challenged the concept of the superiority of centered dual electrodes by showing that one single quadripolar electrode in midline has the ability to stimulate the axial low back. These were acute observations, and no data exist as to the long-term behavior of single versus dual electrodes. The advent of tripole electrodes and the ability to steer current has made it more plausible to aim for low-back paresthesia. Further, flanking the 
cathode by lateral anodes also appears, in theory at least, to raise the discomfort threshold. ${ }^{33}$

Marchand et al. ${ }^{34}$ conducted a prospective, randomized controlled study examining patients with at least one prior surgery for chronic back pain secondary to trauma. All the patients used a SCS and acted as their own control. Although a small trial $(n=8)$, pain scores were significantly reduced with SCS, compared with placebo stimulation.

Longitudinal studies by North et al. ${ }^{35}$ showed that, in patients with postsurgical lumbar arachnoid or epidural fibrosis without surgically remediable lesions, SCS is superior to repeated surgical interventions on the lumbar spine (for back and leg pain) and to dorsal ganglionectomy (for leg pain). That study comprised 50 patients with a postlaminectomy syndrome who averaged 3.1 operations prior to SCS implantation. Successful outcome ( $\geq 50 \%$ pain relief and patient satisfaction with the result) was obtained in $53 \%$ of patients at 2.2 years. A systematic review of the literature was conducted by Turner et al., ${ }^{36}$ reviewing a total of 41 articles from 1966 to 1994 that met their criteria. It was noted that for $\sim 50-60 \%$ of patients with postlaminectomy pain, $>50 \%$ pain relief was attained from the use of SCS. In 1996, Burchiel et al. ${ }^{37}$ conducted a prospective multicenter study with 1-year follow-up and also reported $55 \%$ successful stimulation. Medication usage and work status were not changed significantly.

North et al. ${ }^{38}$ also conducted a prospective study randomizing patients with failed back surgery syndrome to either repeat their back surgery, or undergo SCS surgery. Patients were allowed to cross over after 6 months. Ten of 15 patients crossed over from back surgery to SCS, whereas only 2 of 12 patients crossed over from SCS to back surgery.

Studies do not routinely differentiate between axial back and leg pain. What recent data there are on back pain remain inconclusive. In our experience, most implanting physicians have found that SCS is far more effective for radicular pain than for axial low back pain.

\section{Angina}

The role of SCS in the management of refractory angina pectoris seems to be a very promising. There are well-documented reports in the literature of uniformly good results in the relief of anginal pain. ${ }^{39-43}$ Furthermore, the results have been maintained in long-term follow-up and have been substantiated also by a reduction in the intake of nitrates. Notably, other findings have supported the evidence that SCS has effects that go beyond pain relief. The observations that there is less ST segment depression and that the exercise capacity, the time-to-angina, and the recovery time all improve with stimulation suggest that there may be a reduction in ischemia. In a positron emission tomography study, a redistribution of myocardial flow in favor of ischemic parts of the myocardium has been demonstrated as a long-term effect of spinal cord stimulation, both at rest and after pharmacologic stress induction. ${ }^{44}$

Nienke et al. ${ }^{45}$ conducted a prospective study on quality of life changes in patients with refractory angina pectoris implanted with SCS. They found that both the pain and the health aspects of quality of life improved significantly after 3 months of SCS. After 1 year of SCS, social, mental and physical aspects of quality of life also were found to be improved.

Hautvast et al. ${ }^{46}$ implanted SCS in patients with stable angina pectoris and randomized them. One group's stimulator remained inactivated; the other group was instructed to use the stimulator three times a day for 1 hour and with any angina attack. At 6 weeks, compared with controls, the treatment group had increased exercise duration and time to angina, and decreased anginal attacks and sublingual nitrate consumption. Also observed was a decrease in ischemic episodes on EKG, as well as a decrease in observed ST segment depressions on exercise EKG. There was an increase in perceived quality of life and a decrease in pain. It was shown that a placebo effect from surgery in the treatment group was unlikely because all patients had implantation surgery at baseline.

In the ESBY study, Mannheimer et al. ${ }^{47}$ randomized 104 patients accepted for coronary artery bypass graft $(\mathrm{CABG})$ to receive either CABG $(n=51)$ or SCS $(n=$ 53). This study demonstrated that patients randomized to SCS showed a greater than $30 \%$ improvement in Nottingham Health Profile NHP scores, compared with baseline, which was significant and comparable to the improvement shown by patients randomized to CABG. ${ }^{48}$ These results were consistent on follow-up after 4 years. Notably, the 5-year mortality of $27.9 \%$ in the ESBY study was similar between those receiving SCS and those who received CABG, with no difference in the percentage of cardiac deaths. The ESBY study showed that cardiac events were similar across the groups, but that there were significantly more cerebrovascular events observed in the CABG group. Both groups experienced a significant reduction in both the number of angina attacks and the consumption of nitrates. There was no significant intergroup difference regarding these parameters. In another prospective study of 104 patients who underwent SCS implantation for refractory angina pectoris, there was a significant decrease in angina episodes at rest, angina episodes with activity, and total angina episodes. ${ }^{49}$

DeJongste et al. ${ }^{41}$ randomized 17 patients with angina to an active treatment group with SCS implantation and a control group. The control group was followed for 2 months and then received SCS implantation. Both groups were followed for a total of 12 months. This study also revealed a significant reduction in the incidence of angina attacks and in the consumption of nitrates $(p<$ $0.05)$. 
Five studies are reported to be prospective but without matched controls. ${ }^{45,50-53}$ Each of these revealed significant benefit from spinal cord stimulation. The benefit indices ranged from reduction in angina attacks and decreased nitrate consumption to decreases in NYHA grade and improvement in NHP grade.

The concern whether stimulation can conceal an acute myocardial infarction was addressed by Andersen et al. ${ }^{54}$ They reported on 3 out of 45 patients treated with SCS for anginal pain who survived myocardial infarction. All three patients noticed the pain to be different and unrelieved with SCS and all patients correctly guessed that the pain was due to myocardial infarction. The authors concluded that SCS for treatment of anginal pain does not seem to conceal acute myocardial infarction. Anderson followed this up in 1994, further concluding that neurostimulation does not conceal the pain of an acute myocardial infarction. ${ }^{55}$ In particular, SCS reduced the severity of anginal attack but did not suppress conduction and perception of the cardiac pain signals that act as alarm signals of cardiac distress. ${ }^{56}$ Murray et al. ${ }^{57}$ have shown that SCS for refractory angina is effective in preventing hospital admissions without masking ischemic symptoms or leading to silent infarction.

The mechanisms of action of SCS are unclear. There may be homogenization of myocardial blood perfusion with SCS, and that this reduces myocardial ischemia. ${ }^{44,58}$ Another study has demonstrated that SCS improved heart muscle lactate metabolism and oxygen demand and blood flow in the coronary sinus. ${ }^{59}$ Other studies show that SCS does not affect variability in heart rate or cardiac arrhythmias. ${ }^{40,60,61}$ Hautvast et al. ${ }^{62}$ found no significant changes in heart rate variability after 6 weeks and concluded that heart rate variability via autonomic modulation may not be the explanatory mechanism of action.

Because the relation between pain and myocardial ischemia has not been fully clarified, we do not know whether the pain relief is due to direct depression of nociceptive signals in the spinal cord or whether there is secondary gain from a reduction in the ischemia. ${ }^{63,64} \mathrm{~A}$ significant amount of work by Foreman ${ }^{18}$ has shown that dorsal column stimulation inhibits the activity of spinothalamic tracts cells evoked by activation of the cardiac sympathetic afferents or by intracardiac bradykinin. On the other hand, the effects of stimulation might be equivalent to those of a sympathectomy and may act by producing a prolonged inhibition of the hyperactive sympathetic system. Such mechanism has been shown experimentally in the rat by Linderoth et al. ${ }^{20}$

The most appropriate electrode location for the treatment of angina pectoris is most likely the lower cervical and upper thoracic region, although some have reported successful higher cervical placements. ${ }^{47}$ Another consideration is continuous versus cyclical use of SCS. In practice, patients using SCS for angina pectoris often use low-intensity stimulation for several hours per day for prophylactic purposes. ${ }^{65}$ Recently, a randomized control study demonstrated improvement in functional status and symptoms in treatment arms with conventional or subthreshold stimulation, compared with a low-output placebo treatment arm. ${ }^{66}$ This is the first blinded study in which stimulation below the sensory threshold for paresthesia demonstrated therapeutic efficacy, thus eliminating the possibility of a placebo effect.

The success of the procedure ultimately will be determined by cardiologists. The question arises of the actual number of patients who continue, despite all treatment modalities (including coronary bypass), to have symptoms of such magnitude as to require a spinal cord stimulator. As with the indication of SCS for peripheral vascular disease, European physicians have demonstrated a substantially greater interest in the modality than U.S. physicians. Similar to other applications, a substantial amount of data from well-controlled clinical studies will be necessary before the SCS procedure for angina pectoris will be fully endorsed by the medical community in the United States.

\section{Chronic critical limb ischemia and pain}

Cook and Weinstein ${ }^{67}$ were the first to suggest, in 1973, that the indications for SCS might extend beyond intractable pain control. They observed a group of patients with multiple sclerosis who underwent SCS to treat their chronic pain. Unexpectedly, the patients experienced not only pain relief but also an improvement in mobility and in sensory and bladder function. Cook et al. ${ }^{68}$ noted apparent improvement in lower limb blood flow, and subsequently used SCS in patients whose primary problem was peripheral vascular disease (PVD). He demonstrated relief of rest pain, increased skin temperature, improved plethysmographic blood flow, and healing of small cutaneous ulcers. Subsequently, Meglio et al. ${ }^{69}$ in 1981 reported pain relief and ulcer healing in a patient with advanced peripheral arterial insufficiency. In 1988, Jacobs et al. ${ }^{70}$ published clinical evidence that SCS improved the microcirculation as measured by capillary microscopy.

Klomp et al. ${ }^{71}$ randomized 120 patients with critical painful limb ischemia to receive either best medical therapy alone or SCS in conjunction with best medical therapy. At a mean follow-up of 19 months, there was no significant difference in pain score improvement between the two groups. Conversely, from a similar study in which 51 patients were randomized to receive either oral medication alone or SCS with oral medication, Jivegård et al. ${ }^{72}$ reported a significant improvement in pain scores of the SCS-treated group, compared with the non-SCS group $(p<0.01)$.

Four reported studies without matched controls reveal an overall success rate of $78 \%(n=271) .{ }^{73-78}$ Analysis of seven retrospective studies found an overall success 
rate of $76 \%(n=308) .{ }^{29} \mathrm{~A}$ review of the European literature shows that $70-80 \%$ of patients achieved significant $(>75 \%)$ pain relief, and many other large studies have been reported on the long-term results of SCS on pain and ulcer healing. ${ }^{73-78}$

In a prospective randomized trial by Guarnera et al., ${ }^{79}$ comparison of the effectiveness of SCS versus distal arterial reconstruction demonstrated a more favorable outcome with SCS (72\%) than with distal arterial reconstruction $(40 \%)$. In a Cochrane review ${ }^{80}$ looking at six studies of SCS versus conservative treatment, it was determined that limb salvage after 12 months was significantly higher in the SCS group, and that significant pain relief occurred in both treatment groups but was more prominent in the SCS group.

The mechanisms of action are unclear. The most likely mechanism responsible for increased blood flow in subjects with peripheral vascular disease is inhibition of the sympathetic system. This phenomenon occurs within the spinal cord at the local level and is not related to antidromic activation of afferent fibers. Suprasegmentary influence on medullary vegetative centers does not need to be invoked. The possible role of locally released vasoactive peptides awaits elucidation. Whether the effects on pain and blood flow are due to the same mechanisms is unknown, although some evidence suggests that pain relief is secondary to the microcirculatory changes. Multiple mechanisms may be operating simultaneously. ${ }^{81-85}$

\section{Abdominal and visceral pain syndromes}

Approximately $20 \%$ of the population in the United States have abdominal pain. There are many etiologies for abdominal pain, including gastrointestinal, genitourinary, musculoskeletal, and nervous system. Treatment modalities have included cognitive-behavioral, physical, and pharmacological therapies. Other more invasive therapies include celiac plexus blocks and celiac ganglia destruction. Some studies have demonstrated some localization in the spinal cord for visceral pain secondary to malignancy. Midline myelotomy through the dorsal columns at the level of T10 has shown success in eight patients with refractory pelvic cancer pain. This was also demonstrated in animal studies, in which dorsal column activity was observed in pelvic visceral nociception. ${ }^{86}$

Initially, there was lack of evidence for the application of spinal cord stimulation for visceral and somatic pain, secondary to the belief that nociceptive pain could not be modulated via stimulation. Several initial studies have since demonstrated the benefit of SCS in abdominal visceral disease. Ceballos et al. ${ }^{87}$ demonstrated reduction in pain scores and decrease in narcotic use in a patient treated for mesenteric ischemia. Trial stimulation was 13 days, with implantation followed for 12 months and the electrode placed at T6. The patient had only two small pain recurrences in that period, one of which was when the stimulator was stopped. Krames and Mousad ${ }^{88}$ de- scribed a patient treated for irritable bowel syndrome who was developing escalating pain and diarrhea. Although the pain treatment eventually required intrathecal therapy, there was a significant reduction in the amount of diarrhea. The patient underwent a 2-week trial and subsequently had implantation at the T8 level. In the first 6 months, there was a subjective decrease in pain from $9 / 10$ to $2 / 10$, with only two diarrheal episodes and significant reduction in pain medications. There was some return of pain after 10-month follow-up, requiring increase in pain medications, but the significant reduction in diarrhea remained.

In the largest series to date, Khan et al. ${ }^{89}$ reported on nine patients with refractory abdominal pain. Five of the nine patients had nonalcoholic pancreatitis, three had presumed abdominal wall neuromas from frequent abdominal surgery, and the last had postsplenectomy pain after trauma. At 6- to 8-month follow-up, with placement of the leads at the T5-7 level, all patients had a significant improvement in VAS scores, as well as decreased narcotic use.

Tiede et al..$^{90}$ described treatment of refractory abdominal pain in two patients. Both patients had a significant history including multiple abdominal surgeries and failed conservative measures. Each patient had an element of postprandial abdominal pain with associated nausea and vomiting. In both patients, the leads were placed at the T2 level with significant improvement in pain, decreased narcotic use, and increased functioning, such as return to work. Kapur et al. ${ }^{91}$ recently described relief of abdominal pain associated with colchicine intolerance or resistance in patients with familial Mediterranean fever, with placement of the electrodes at the lower thoracic levels.

More recently, studies have looked at the treatment of visceral pelvic pain with reference to the dorsal columns and spinal cord stimulation. Kapural et al. ${ }^{92}$ reported on the value of neurostimulation for chronic visceral pelvic pain in six women with the diagnosis of long-standing pelvic pain. These patients had a history of endometriosis, multiple surgical explorations, and dyspareunia. At an average follow-up of 30 months, there was a significant decrease in the VAS score and an average of $>50 \%$ pain relief, with a decrease in opiate use.

Visceral innervation follows the embryologic origin and location of the viscera and is arranged in viscerotomes, analogous to cutaneous dermatomes. ${ }^{93}$ The viscera obtain their innervation via the sympathetic and parasympathetic pathways. The parasympathetics carry their afferents to anterior and posterior vagal trunks and are therefore not as amenable to spinal cord stimulation. The sympathetics carry nociceptive information from the viscera to spinal nerve roots, which makes them a more viable target. The sympathetic afferents in the lower six thoracic and the upper three lumbar spinal segments have been shown to transmit painful impulses from the viscera. ${ }^{94}$ 


\section{RELEVANT ANATOMY}

Understanding the somatotopy of the spinal cord is paramount in understanding the technical aspects of implantation. A basic tenet of SCS is to create an overlapping of paresthesia and pain region. To this end, correlation of the somatotopy and the level of the spinal cord is necessary. Barolat et al. ${ }^{95}$ have published extensively on the mapping of the human spinal structures. A database was created to suggest areas of sensory response to dorsal spinal cord stimulation.

High cervical regions such as $\mathrm{C} 2$ can cover the posterior occipital region, and occasionally the lower jaw. C2-4 stimulation will provide coverage of the shoulder, and stimulation in the lower cervical region (e.g., C5-6) will provide coverage for the entire hand. To cover the anterior chest wall or the axilla, an electrode toward C7 is necessary.

More commonly, an implanter will seek to cover the lower extremities. Lateral placement at T11-12 will cover the anterior thigh, and placement at T11-L1 can cover the posterior thigh. Coverage of the foot as a whole can be achieved along these same areas, but it becomes more difficult to cover the sole of the foot. Alternatively, for coverage of the sole of the foot, a patient may require insertion on the lumbar L5 or S1 nerve roots. Low back pain is very difficult to cover, because midthoracic stimulation can affect the chest and abdominal wall. In the authors' experience, the best localization has been with midline placement at T8-9.

Finally, most patients prefer stimulation of the dorsal column from electrodes closer to the midline. Laterally, placed thoracic electrodes are more likely to stimulate the thoracic nerve roots and result in painful stimulation.

\section{EQUIPMENT: ELECTRODES AND PULSE GENERATORS}

The implanting physician should become familiar with the various implantable technologies available for SCS. These include trial percutaneous electrodes, permanent percutaneous electrodes, permanent plate electrodes, totally implantable rechargeable and nonrechargeable pulse generators (IPG), and RF-driven pulse generators.

\section{Percutaneous electrodes}

Percutaneous electrodes (or wire electrodes) are particularly appealing. Percutaneous electrodes can be inserted without much dissection and offer a substantial advantage when one is performing a trial to assess candidacy for a permanent implant. After the trial period, the temporary percutaneous electrode can easily be removed in the implanting physician's office. During implantation, these electrodes can be advanced over several segments in the epidural space, allowing testing of several spinal cord levels to assess for optimal electrode position.
Contemporary percutaneous electrodes are slim electrodes, only a few millimeters in diameter, that contain four or eight contacts with various spacings (referred to as either quadripolar or octapolar electrodes). Choosing the particular electrode entails deciding how many segments of the spinal cord are to be covered, with larger spacing allowing broader coverage. Alternatively, closer spacing allows better steering and electric field shaping. Additionally, multiple parallel electrodes and different configuration matrices can be constructed, which can create extremely focused electrical fields. The general trend is to use one or two quadripolar electrodes for limb pain and one or two octapolar electrodes for axial pain. Even insertion of three electrodes is being explored for better steering of current. ${ }^{33}$ These percutaneous electrodes also come in varying lengths, and these differ by manufacturer. At times, extensions cables are necessary to bridge the distance from the spinal entry point to the pocket in which the battery will reside.

\section{The technique of percutaneous implantation}

The patient is positioned in a comfortable prone position on a fluoroscopy table. A pillow underneath the abdomen may create some kyphosis, which can facilitate electrode insertion.

The level of electrode insertion is guided by several factors. A fundamental consideration is that several centimeters of the lead have to lie in the epidural space to assure maximal stability of the electrode and minimize unwanted migration. To assure this, insertion must take place at least two spine segments below the desired target. For cervical placement, one must be aware of the cervical cord enlargement and, when possible, electrode insertion should be performed below the T1-2 level. Some surgeons advocate upper thoracic placement of percutaneous electrodes through a limited spinous process removal, to minimize the potential risk of spinal cord damage. For practical purposes, implantation for low-back problems necessitates electrode insertion at T12-L1 or L1-2 and implantation for an upper extremity target requires insertion at T2-3 or T3-4.

The fluoroscopy equipment must be ready to function in both the anterolateral and lateral planes at the time of needle insertion. The Tuohy needle is inserted with as shallow an angle as possible. In the thoracic area, this can be accomplished with either a midline or paramedian approach; in the upper lumbar area, a paramedian approach is required. A steep angle increases the risk of electrode fracture.

Several methods are available to identify the epidural space. Tactile feedback is important, but cannot be solely relied upon. The most approach is the loss of resistance method using a low-friction glass syringe. We prefer to inject a small amount of air. Fluid injected in the epidural space may later be aspirated through the needle and give the false impression of being in the subarachnoid space. 
After multiple passes at one spine level have been performed, the loss of resistance method may lose its reliability. Inserting a Seldinger wire through the needle can provide invaluable information as to the degree of penetration into the spinal canal. If the needle tip is in the interspinous ligament and has not penetrated the ligamentum flavum, the wire cannot be advanced. The wire can be advanced only if the needle tip is in the paraspinal muscles or within the spinal canal. The pattern of advancement and the location of the wire under fluoroscopic imaging can further clarify its position.

Once the electrode is in the spinal canal, one has to be certain that it is positioned in the epidural space and not within the subarachnoid space. Even though it may seem obvious and easily recognizable, this can become a very difficult task and require multiple attempts at needle placement. This is particularly true if the arachnoid has been previously pierced and cerebrospinal fluid has escaped and pooled in the dorsal epidural space. In the subarachnoid space, much less resistance is encountered when moving the electrode, particularly for lateral movements. The wire seems almost to be floating and undergoes large shifts of direction, whereas in the epidural space movements are more discrete and obtained only with specific manipulations. The same type of wire or electrode movement can, however, be experienced epidurally if the dural sac has significantly collapsed secondary to loss of cerebrospinal fluid. Electrical stimulation will clarify the position, because a subarachnoid placement can elicit motor or sensory responses at much lower thresholds than epidural placement.

When the epidural space is satisfactorily identified, the electrode is gently inserted under fluoroscopic guidance in the anteroposterior view. Removal of the electrode once it has been inserted through the tip of the needle has to be accomplished without stripping the insulation on the lead. If the electrode does not slide with minimal resistance, the needle and the electrode should be removed together. Every time the electrode is withdrawn through the needle, it should be inspected for minute breaks in the insulation, which would necessitate its disposal. Alternatively, a sleeve can be inserted over the guide wire in the epidural space. The guide wire is then removed and the electrode is inserted through the sleeve. This reduces the risk of shearing the electrode during manipulation. The electrode is then steered in the epidural space to the desired location. Should the targeted location prove to be less than two spinal segments from the electrode insertion, the electrode should be withdrawn and repositioned at a more caudal level.

Frequently, the electrode curves around the dural sac and ends in the ventral epidural space. In the anteroposterior projection this might be indistinguishable from a proper midline dorsal location. A gentle lateral curve of the electrode shortly after its entry in the epidural space should arouse suspicion that it is directing ventrally

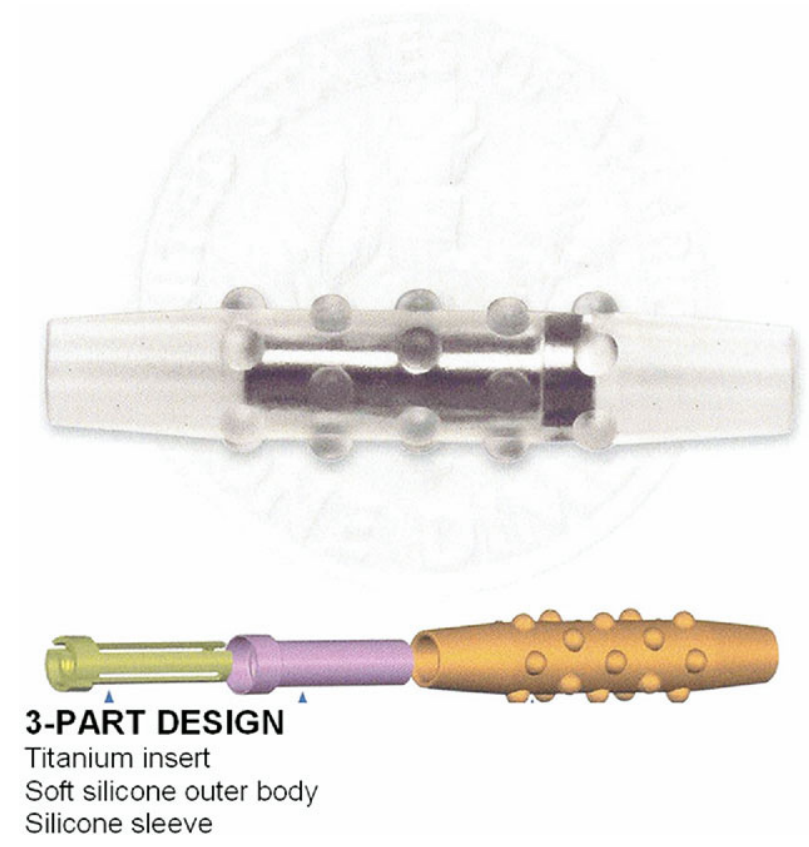

FIG. 1. Titan anchor (Medtronic, Minneapolis, MN; used with permission).

around the dural sac. Absolute confirmation of the ventral location arises from the stimulation generating violent motor contractions or observation in the lateral plane that readily discloses the anterior position of the electrode tip. If more than one electrode is inserted, it is wise to insert the other needles before inserting the electrodes. Needle insertion might shear an already implanted electrode. Besides, it is often possible to insert two electrodes simultaneously and advance them synchronously in the epidural space while maintaining their relative position and spacing.

Once in place, the electrode must be secured to the interspinous ligament to minimize dislodgment. An Xray image is obtained to document electrode level and position. We have found that securing loops at the electrode insertion site serves to relieve the strain and reduces migration during bending. Frequently, anchors are used to secure the electrode and the implanter should remember that anchoring is a two-step process: first securing the anchor to the electrode and then securing the anchor to the fascia. At present, there is only one anchor available that can be detected on radiographs: the TITAN anchor (Medtronic, Minneapolis, MN) (FIG. 1).

A major disadvantage that has been cited with percutaneous electrodes is their tendency to migrate. This is related to their inherent flexibility, necessary for insertion through a Tuohy needle, and to their cylindrical shape, which does not prevent migration even months after implantation. Some percutaneous electrodes require a stiffening stylet for introduction. Also, percutaneous electrodes are less energy efficient than plate electrodes. The electrical current is distributed circumferentially around the electrode and is expected to result in greater 


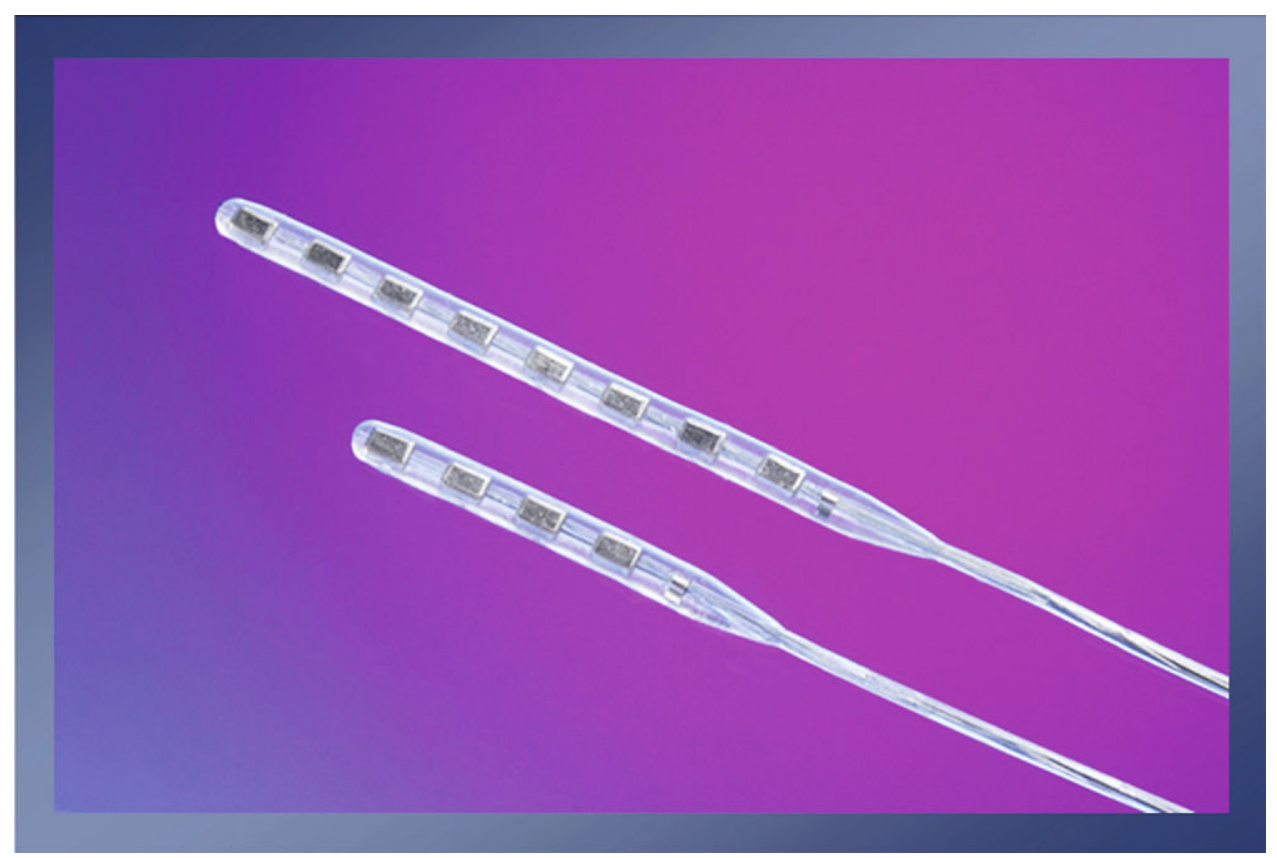

FIG. 2. Slim-line electrode (ANS-Advanced Neuromodulation Systems, Plano, TX; used with permission).

shunting of current. Patients with percutaneous leads also describe a greater positional variance in their paresthesia.

Recently, Advanced Neuromodulation Systems (ANS, Plano, TX) has introduced a slim-line plate-type electrode that can be inserted percutaneously. The broader electrode base provides a surface such that fibrosis should lessen the risk of caudal electrode migration. The slimmer profile of the electrode might also have advantages in the cervical spine, where spinal cord compression could be an issue. Finally, the design emulates that of a miniplate lead, in that the contacts are on one side with the other side being insulated. This will be more energy efficient than a percutaneous lead, which allows delivery of current circumferentially (FIG. 2).

\section{Plate electrodes}

Plate-type electrodes (also known as ribbon electrodes, paddle electrodes, or laminotomy electrodes) require a surgical procedure, laminotomy, and implantation under direct vision. ${ }^{28}$ Implantation under direct vision may be safer in the upper thoracic and cervical areas, where there is a risk of damaging the spinal cord with the large-bore Tuohy needle. Most implants can be done through a skin incision between 2.5 and $4 \mathrm{~cm}$ long, depending on the size of the patient and spinal anatomy. The amount of bony removal is usually minimal.

Multiple arrays or different electrode configurations can be also constructed with plate electrodes. The main advantage of plate electrodes lies in their more inherent stability in the dorsal epidural space and lesser propensity to migrate. Some preliminary data by North et al. ${ }^{96}$ also suggest a broader stimulation pattern and lower stimulation requirements with plate electrodes. Plate electrodes are more energy efficient in delivering elec- trical stimulation. We advocate plate electrodes as the only option in the case of previous spine surgery at the implant levels.

Plate electrodes come in many sizes, shapes, spacing, and configurations. There are single-column and dualcolumn electrodes. As with percutaneous leads, there are varying lengths and shapes (such as curved leads and hinged leads), all designed to help facilitate insertion and tailor the electrode selection to the patient.

There is some literature describing the advantages of plate leads. North et al. ${ }^{96}$ have compared plate and percutaneous electrodes. Laminectomy electrode placement, although more invasive than percutaneous placement, yielded significantly better clinical results in patients with failed back surgery syndrome at up to 3-year follow-up. Clinical success was defined as $\geq 50 \%$ pain relief and patient satisfaction with treatment. Secondary outcome measures were ability to perform various activities of daily living, neurological function, and analgesic use.

There is some theoretical evidence that shaping of the electrical field is possible with even more complex electrode arrays. Holsheimer et al. ${ }^{97}$ concluded that the transverse tripolar system enabled finer control of paresthesia. Electrical field steering could change the paresthesia area completely. When the transverse tripolar configurations are used, the threshold for stimulation of dorsal roots is higher, compared with the dorsal column threshold. This results in a wider therapeutic range, wider paresthesia coverage, and a greater probability to fully cover the painful area with paresthesia.

One must bear in mind that the increasing number of contacts brings with it a significant increase in power consumption. The complexity of programming rises in 
even a greater magnitude. With 2 contacts, the total number of configurations possible are 8 , with 4 contacts, 64; and with 8 and with 16 contacts, it increases exponentially to reach a number in the millions.

Two basic positions can be used for the laminotomy and implantation: prone or semilateral. The prone position allows a more intuitive understanding of the spatial relations and is one that more surgeons are familiar with. In this position, however, it can be difficult to obtain adequate sedation for the surgical exposure and also maintain the airway. In the semilateral position, the patient lies comfortably in a park bench-type position, allowing access to both the spine and the flank, abdomen, or buttock for the implant of the pulse generator. The patient is asked to place him or herself in the most comfortable position. If the pain is predominantly on one side, the patient is asked to lie on the less affected side. In this position, airway management is safer than in the prone position, and the anesthesiologist is more comfortable in keeping the patient deeply sedated. Given the variable degree of rotation of the body, however, it can be difficult for the surgeon to determine the location of the midline. This might constitute a significant problem in the cervical area.

The planned level is localized either with fluoroscopy or with a plain X-ray with metallic markers placed on the skin at the level of the planned incision. In a thin individual, the incision is $\sim 1$ inch in length $(\sim 2.5 \mathrm{~cm})$; even in large individuals, the incision seldom needs to be more than 2 inches long. Different considerations apply if one is implanting through a level previously operated on.

For cervical placement, the patient is placed in the semilateral position with the neck slightly flexed. Even with a short skin incision, one can reach three to four levels by extending the inside dissection and stretching the skin edges with a Gelpi retractor. The neck should be flexed, but not excessively rotated laterally; even though some neck rotation is inevitable, extreme rotation substantially increases the level of difficulty.

Subperiosteal dissection is usually limited to the upper half of the spinous process inferior to the addressed ligamentum flavum and to the whole spinous process superior to it. Parts of the superior spinous process are incrementally removed until the ligamentum flavum is exposed. In the lower thoracic or upper lumbar area, this usually results in removal of the inferior one third of the spinous process. In the midthoracic area, due to the acute angle and significant overlapping of the spinous processes, the whole spinous process must be removed. After removal of the ligamentum flavum, the electrode or electrodes are inserted in the dorsal epidural space; the electrode position is then confirmed with fluoroscopy and test stimulation is performed with the patient awakened and able to report where he or she is feeling paresthesias.

Alternatively, the electrode can be placed with the patient fully in the prone position under general anesthesia. Fluoroscopy should be used to identify the same level where the active contacts were placed during the trial. A laminotomy is performed approximately one level below this point, to allow the plate electrode to reach up to the intended level. After placing the electrode, intraoperative stimulation with electromyographic correlation will be able to detect stimulation in the extremity and lateralization of the electrode. We stimulate the electrode with $5-\mathrm{Hz}$ stimulation at $>310 \mu$ s pulse width and ramp up the amplitude until electromyographic signal changes are detected. Bilateral extremity stimulation suggests midline placement, and early root onset implies too lateral a placement. We have had instances in which the physiological midline differed from the anatomic midline; in such cases, we are more apt to rely on the intraoperative physiology. When treating axial symptomatology, the lead is placed to straddle the midline. For patients with unilateral pain, the lead is placed so that one array is on the side of the pain and the other is on the midline.

\section{Rechargeable and nonrechargeable pulse generators and radio-frequency receivers}

Electrical stimulation consists of rectangular pulses delivered to the epidural space through an implanted electrode via a power source. Two basic types of systems are currently available: an Internal Pulse Generator (IPG; also called the battery) or a radio-frequency (RF) coupled pulse generator with an implantable receiver. The latter has largely fallen out of favor, because of the inconveniences of the external power source. The advent of the totally implantable, rechargeable pulse generator has surmounted the power requirement issues, which were previously the real RF advantage (FIG. 3).

The totally implantable pulse generator contains a lithium battery. Activation and control occur through an external transcutaneous telemetry device. The IPG can be turned on and off through a small controller which the patient can carry. The controller also allows some control over the stimulation parameters. More extensive control of the unit can be achieved through a small portable unit that can be programmed by the physician. Life span of the battery varies with usage and with the parameters used (i.e., voltage, rate, and pulse width). Most patients can expect the battery to last, under average usage, between 2.5 to 4.5 years. Available lithium-powered pulse generators allow stimulation with fine resolution increments of $0.05 \mathrm{~V}$ and with varying rates and pulse widths. Replacement of the battery requires a surgical procedure, one that is usually performed on an outpatient basis.

A particular IPG is selected based on many variables. From a practical standpoint, the first and foremost reason might be the size of the patient. Although larger batteries will have longer life, the site of insertion of the IPG (either the buttock, abdomen, or the subclavicular re- 

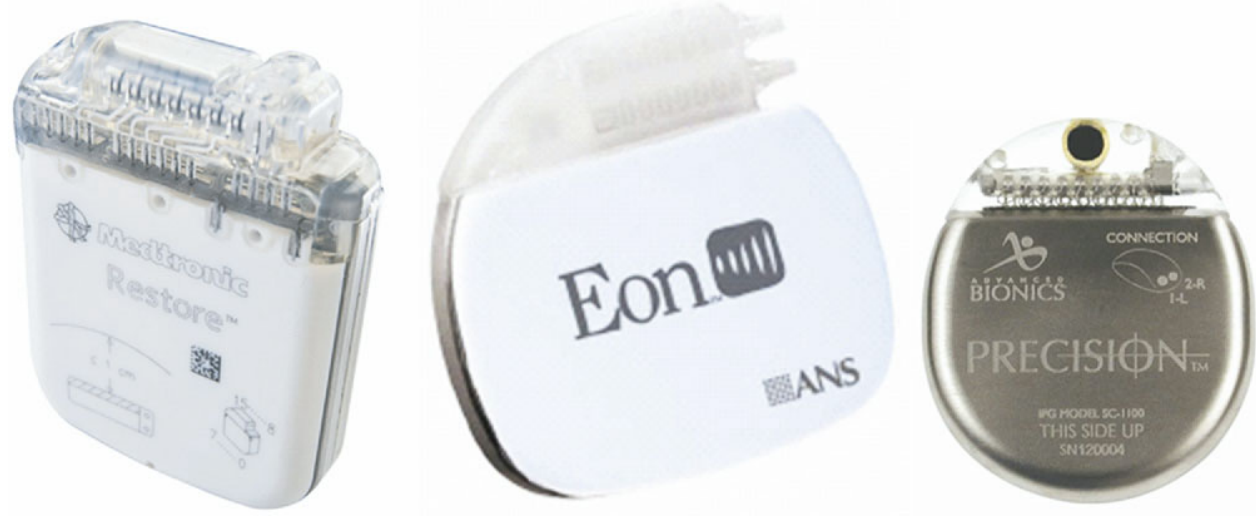

FIG. 3. Rechargeable batteries.

gion) is often the source of significant patient complaint. We prefer to implant the IPG in the buttock because of ease in tunneling from the electrode insertion. Furthermore, with a patient placed prone for electrode insertion, there is no repositioning required to reach the buttock region. We identify three bony prominences (the posterior superior iliac crest, the greater trochanter of the femur, and the apex of the iliac crest) and implant the IPG in the lateral aspect of this triangle (FIG. 4).

RF-driven systems consist of a passive receiver, implanted subcutaneously, and a transmitter that is worn externally. An antenna applied to the skin in correspondence of the receiver is connected to the transmitter, which sends the stimulation signals transcutaneously. For the system to function, the transmitter has to contain charged alkaline batteries and the antenna must make adequate contact with the receiver. This requires the patient to wear the external system in order to receive the stimulation. RF-driven systems can deliver stimulation with rates up to $1400 \mathrm{~Hz}$, and can be customized to deliver high power levels.

RF systems involve the inconvenience of having to wear the antenna and the radio receiver. The problem might go beyond pure inconvenience for individuals who have handicapped motor function in the upper extremities and cannot properly go through all the steps required to make the external unit function properly. Other patients, particularly those who have reflex sympathetic dystrophy (RSD), may not tolerate an antenna taped to the skin. Obtaining adequate contact of the receiver with the skin may be difficult secondary to swelling at the site.

The equipment cannot be worn while swimming or showering, and severe perspiration, as with exercise and physical therapy, might make proper contact of the an-

\section{Lateral Femur}

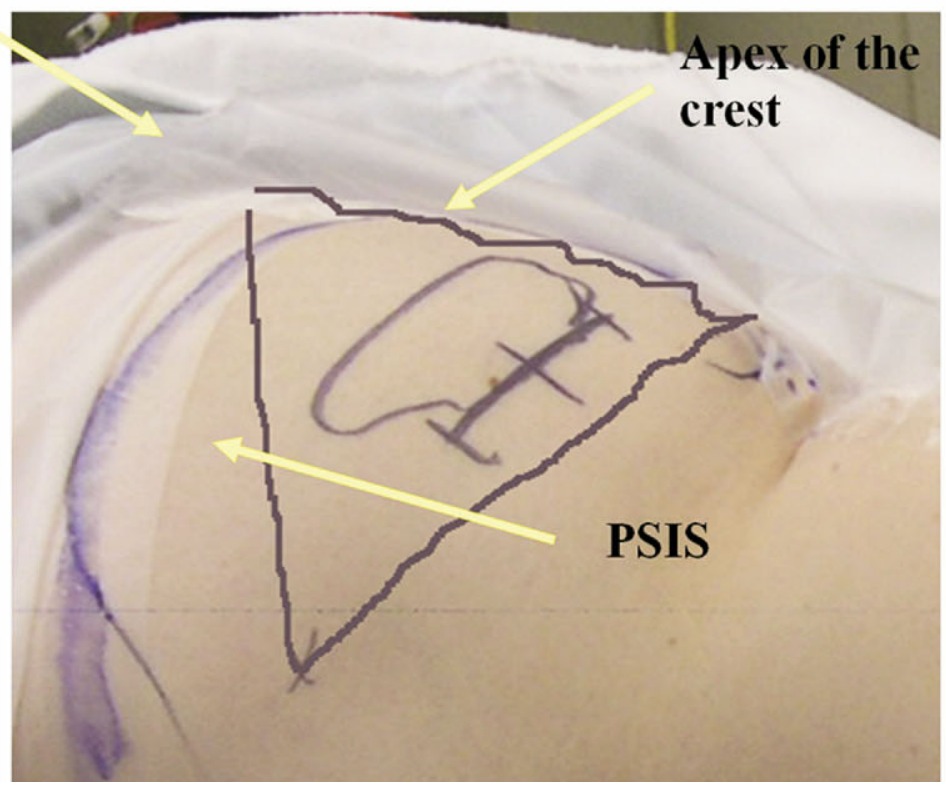

FIG. 4. Battery placement. In our technique for the placement of the battery in the buttock, the bony prominences are marked: the greater trochanter (lateral femur), the apex of the iliac crest, and the posterior superior iliac spine (PSIS). A triangle is created, and the battery incision is made parallel to the top rung of the triangle. 
TABLE 1. Specifications for Three Rechargeable Batteries

\begin{tabular}{|c|c|c|c|}
\hline & Precision Plus & Restore Advanced & Eon \\
\hline Volume, $\mathrm{cm}^{3}$ & 22 & 39 & 42 \\
\hline Dimensions, mm & $55 \times 45 \times 11$ & $65 \times 49 \times 15$ & $59 \times 58 \times 16$ \\
\hline Weight, g & 36 & 72 & 75 \\
\hline Stimulation Output & Multiple current sources & Constant voltage & Constant current \\
\hline Amplitude, $\mathrm{mA}$ or $\mathrm{V}$ & $0-20 \mathrm{~mA}$ & $0-10.5 \mathrm{~V}$ & $0-25.5 \mathrm{~mA}$ \\
\hline Frequency, $\mathrm{Hz}$ & $2-1200$ & $2-130$ & $2-1200$ \\
\hline Pulse Width, ms & $20-1000$ & $60-450$ & $50-500$ \\
\hline Battery Capacity, mA h & 200 & 300 & 325 \\
\hline Recharging Type & Cordless & Cordless & Connect to Outlet \\
\hline $\begin{array}{l}\text { Wireless Communication } \\
\text { Distance, inches }(\mathrm{cm})\end{array}$ & $\leq 30(\leq 76)$ & $\leq 4(\leq 10)$ & not applicable \\
\hline $\begin{array}{l}\text { Maximum Recharge } \\
\text { Depth, cm }\end{array}$ & 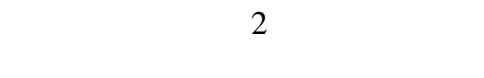 & 1 & 2.5 \\
\hline Manufacturer & $\begin{array}{l}\text { Boston Scientific, Natick, MA } \\
\text { (http://www.bostonscientific.com) }\end{array}$ & $\begin{array}{l}\text { Medtronic, Minneapolis, MN } \\
\text { (http://www.medtronic.com) }\end{array}$ & $\begin{array}{l}\text { Advanced Neuromodulation } \\
\text { Systems, Plano, TX } \\
\text { (http://www.medical.com) }\end{array}$ \\
\hline
\end{tabular}

tenna problematic. Furthermore, the patient has to replace batteries on a regular basis and make sure that proper coupling exists between the antenna and the receiver at all times. What one loses in convenience, however, is gained in power and flexibility. Currently, only RF systems can provide a stimulation rate up to $1400 \mathrm{~Hz}$. This might be beneficial in some patients with neuropathic chronic pain syndromes, ${ }^{98}$ as well as in patients with extrapyramidal motor disorders.

Rechargeable systems have now become available. A Medtronic device, known as the Restore rechargeable neurostimulation system, uses a battery with an estimated 9-year total life span. It takes $\sim 6$ hours to fully recharge the batteries. The Advanced Neuromodulation Systems Eon device has a battery life that is currently estimated at 7 years. The Boston Scientific Precision device has a battery life estimated at 5 year. A detailed comparison of the features of the rechargeable batteries is given in Table 1.

\section{COMPLICATIONS}

With the proper expertise, permanent complications of SCS are rare. ${ }^{70}$ The most serious complication, which is shared with any type of spine surgery, is paralysis or severe neurological deficit. This can occur during spinal cord stimulation procedures, both with percutaneous and plate electrodes. Infection of the implanted hardware has occurred at a $3-5 \%$ rate. ${ }^{99,100}$ Persistent pain at the implant site has been seen in $\sim 5 \%$ of patients. ${ }^{99,100}$ Recalcitrant cerebrospinal fluid leakage has been encountered in a few patients, requiring multiple surgical revisions. Breakage or malfunction of the implanted hardware (particularly the electrodes and the subcutaneous extension cables) or migration of the electrode has been encountered in $\sim 11-45 \%$ of the implanted systems. ${ }^{29}$ Painful stimulation, necessitating either repositioning or removal of the electrode, has also been reported in a number of cases. $^{29}$

\section{CONCLUSIONS}

The treatment of chronic pain remains challenging. Spinal cord stimulation has been performed for more than 30 years, and slow but steady progress with this technology has been made. As the equipment and stimulation parameters are improved, selection criteria have been better defined and are slowly being expanded. More important, experience in the technique and the equipment has made SCS a much more reliable and safe modality. As with all the modalities performed for chronic pain management, its results are favorable. It is important to remember that the goal of neurostimulation is to reduce pain, rather than to eliminate pain. SCS has been shown to have a 50\% improvement in pain relief. Very few other invasive modalities can claim this success rate with a few years of follow-up.

Careful follow-up of patients is necessary for successful long-term satisfaction. Equipment-related problems can arise at any time after implantation, such as discomfort at the pulse generator or radio receiver site, electrode breakage or migration, infection, and the like, and an open dialog with patients is vital for the continuing successful implementation of the modality. Spinal cord stimulation has earned a firm and well-established role in contemporary chronic pain management.

\section{REFERENCES}

1. Devulder J, De Colvenaer L, Rolly G, Caemaert J, Calliauw L, Martens F. Spinal cord stimulation in chronic pain therapy. Clin J Pain 1990;6:51-56.

2. Devulder J, Vermeulen H, De Colvenaer L, Rolly G, Calliauw L, Caemaert J. Spinal cord stimulation in chronic pain: evaluation of results, complications, and technical considerations in sixty-nine patients. Clin J Pain 1991;7:21-28. 
3. Racz GB, McCarron RF, Talboys P. Percutaneous dorsal column stimulator for chronic pain control. Spine 1989;14:1-4.

4. Melzack R, Wall PD. Pain mechanisms: a new theory. Science 1965;150:971-979.

5. Shealy CN, Cady RK. Historical perspective of pain management. In: Weiner S, editor.Pain management: a practical guide for clinicians, 5th ed.1998Boca Raton, FL: St. Lucie Press, 7-15.

6. Shealy CN, Mortimer JT, Reswick JB. Electrical inhibition of pain by stimulation of the dorsal columns: preliminary clinical report. Anesth Analg 1967;46:489-491.

7. Hoppenstein R. A device for measuring intracranial pressure. Lancet 1965;1:90-91.

8. Larson SJ, Sances A, Cusick JF, Meyer GA, Swiontek T. A comparison between anterior and posterior spinal implant systems. Surg Neurol 1975;4:180-186.

9. Lazorthes Y, Verdie JC, Arbus L. Anterior and posterior medullary analgesic stimulation, using a percutaneous implantation technic [In French]. Acta Neurochir (Wien) 1978;40:277-283.

10. Dooley DM. Percutaneous electrical stimulation of the spinal cord. Assoc. Neurol. Surg. Bal Harbour, FL, 1975.

11. Waltz JM. Computerized percutaneous multi-level spinal cord stimulation in motor disorders. Appl Neurophysiol 1982;45: 73-92.

12. Coburn B. Electrical stimulation of the spinal cord: two-dimensional finite element analysis with particular reference to epidural electrodes. Med Biol Eng Comput 1980;18:573-584.

13. Coburn B. A theoretical study of epidural electrical stimulation of the spinal cord-Part II: Effects on long myelinated fibers. IEEE Trans Biomed Eng 1985;32:978-986.

14. Coburn B, Sin WK. A theoretical study of epidural electrical stimulation of the spinal cord-Part I: Finite element analysis of stimulus fields. IEEE Trans Biomed Eng 1985;32:971-977.

15. Holsheimer J, Barolat G, Struijk JJ, He J. Significance of the spinal cord position in spinal cord stimulation. Acta Neurochir Suppl 1995;64:119-124.

16. Holsheimer J, Struijk JJ. How do geometric factors influence epidural spinal cord stimulation? A quantitative analysis by computer modeling. Stereotact Funct Neurosurg 1991;56:234-249.

17. Holsheimer J, Wesselink WA. Effect of anode-cathode configuration on paresthesia coverage in spinal cord stimulation. Neurosurgery 1997;41:654-659; discussion 659-660.

18. Foreman RD, Beall JE, Coulter JD, Willis WD. Effects of dorsal column stimulation on primate spinothalamic tract neurons. J Neurophysiol 1976;39:534-546.

19. Linderoth B, Gazelius B, Franck J, Brodin E. Dorsal column stimulation induces release of serotonin and substance $\mathrm{P}$ in the cat dorsal horn. Neurosurgery 1992;31:289-296; discussion 296297.

20. Linderoth B, Stiller CO, Gunasekera L, O'Connor WT, Ungerstedt U, Brodin E. Gamma-aminobutyric acid is released in the dorsal horn by electrical spinal cord stimulation: an in vivo microdialysis study in the rat. Neurosurgery 1994;34:484-488; discussion 488-489.

21. Barolat G, Schwartzman R, Woo R. Epidural spinal cord stimulation in the management of reflex sympathetic dystrophy. Stereotact Funct Neurosurg 1989;53:29-39.

22. Kumar K, Nath RK, Toth C. Spinal cord stimulation is effective in the management of reflex sympathetic dystrophy. Neurosurgery 1997;40:503-508; discussion 508-509.

23. Kemler MA, Barendse GA, Van Kleef M, Van Den Wildenberg FA, Weber WE. Electrical spinal cord stimulation in reflex sympathetic dystrophy: retrospective analysis of 23 patients. J Neurosurg 1999;90(1 Suppl):79-83.

24. Kemler MA, Barendse GA, van Kleef M, et al. Spinal cord stimulation in patients with chronic reflex sympathetic dystrophy. N Engl J Med 2000;343:618-624.

25. Kemler MA, de Vet HC, Barendse GA, van den Wildenberg FA, van Kleef M. Spinal cord stimulation for chronic reflex sympathetic dystrophy: five-year follow-up. N Engl J Med 2006;354: 2394-2396.

26. Oakley JC, Weiner RL. Spinal cord stimulation for complex regional pain syndrome: a prospective study of 19 patients at two centers. Neuromodulation 1999;2:47-50.

27. Calvillo O, Racz G, Didie J, Smith K. Neuroaugmentation in the treatment of complex regional pain syndrome of the upper extremity. Acta Orthop Belg 1998;64:57-63.

28. Ebel H, Balogh A, Volz M, Klug N. Augmentative treatment of chronic deafferentation pain syndromes after peripheral nerve lesions. Minim Invasive Neurosurg 2000;43:44-50.

29. Cameron T. Safety and efficacy of spinal cord stimulation for the treatment of chronic pain: a 20-year literature review. J Neurosurg 2004;100:254-267.

30. Law JD. Targeting a spinal stimulator to treat the "failed back surgery syndrome.' Appl Neurophysiol 1987;50:437-438.

31. Law JD. Spinal stimulation in the "failed back surgery syndrome": Comparison of technical criteria for palliating pain in the leg vs. in the low back. Acta Neurochir 1992;117:95.

32. North R, Kidd DH, Olin J, Sieracki JM, Cutchis PN. Spinal cord stimulation for axial low back pain: single versus dual percutaneous electrodes. In: Neuromodulation for pain and motor disorders. Proc. 4th Int. Congr. Int. Neuromodulation Soc., 16-20 Sept. 1998, Lucerne, Switzerland. San Francisco: International Neuromodulation Society, 1998:212 (abstract). Available at: http://www.neuromodulation.com/ our-neuromodulation-publications.htm.

33. Sharan AD, Miyazawa G, Greenberg J, King G. Selective dorsal column activation with three column electrode arrays using percutaneous and paddle leads. Abstract 737. Presented at Annu Meet Am Assoc Neurol Surgeons (AANS), April 14-19, 2007, Washington, DC. Available at http://www.aans.org [article ID 40855].

34. Marchand S, Bushnell MC, Molina-Negro P, Martinez SN, Duncan GH. The effects of dorsal column stimulation on measures of clinical and experimental pain in man. Pain 1991;45:249-257.

35. North RB, Ewend MG, Lawton MT, Kidd DH, Piantadosi S. Failed back surgery syndrome: 5-year follow-up after spinal cord stimulator implantation. Neurosurgery 1991;28:692-699.

36. Turner JA, Loeser JD, Bell KG. Spinal cord stimulation for chronic low back pain: a systematic literature synthesis. Neurosurgery 1995;37:1088-1095; discussion 1095-1096.

37. Burchiel KJ, Anderson VC, Brown FD, et al. Prospective, multicenter study of spinal cord stimulation for relief of chronic back and extremity pain. Spine 1996;21:2786-2794.

38. North RB, Kidd DH, Piantadosi S. Spinal cord stimulation versus reoperation for failed back surgery syndrome: a prospective, randomized study design. Acta Neurochir Suppl 1995;64:106-108.

39. Augustinsson LE. Spinal cord electrical stimulation in severe angina pectoris: surgical technique, intraoperative physiology, complications, and side effects. Pacing Clin Electrophysiol 1989; 12:693-694.

40. de Jongste MJ, Haaksma J, Hautvast RW, et al. Effects of spinal cord stimulation on myocardial ischaemia during daily life in patients with severe coronary artery disease: a prospective ambulatory electrocardiographic study. Br Heart J 1994;71:413-418.

41. de Jongste MJ, Hautvast RW, Hillege HL, Lie KI; Working Group on Neurocardiology. Efficacy of spinal cord stimulation as adjuvant therapy for intractable angina pectoris: a prospective, randomized clinical study. J Am Coll Cardiol 1994;23:15921597.

42. Mannheimer C, Augustinsson LE, Carlsson CA, Manhem K, Wilhelmsson C. Epidural spinal electrical stimulation in severe angina pectoris. Br Heart J 1988;59:56-61.

43. Sanderson JE, Brooksby P, Waterhouse D, Palmer RB, Neubauer K. Epidural spinal electrical stimulation for severe angina: a study of its effects on symptoms, exercise tolerance and degree of ischaemia. Eur Heart J 1992;13:628-633.

44. Hautvast RW, Blanksma PK, DeJongste MJ, et al. Effect of spinal cord stimulation on myocardial blood flow assessed by positron emission tomography in patients with refractory angina pectoris. Am J Cardiol 1996;77:462-467.

45. Nienke C, Vulink C, Overgaauw DM, Jesserun GAJ, TenVaarwerk IAM, Kropmans TJB, ven der Shans CP, Middel B, Staal MJ, DeJongste MJL. The effects of spinal cord stimulation on quality of life in patients with therapeutically chronic refractory angina pectoris. Neuromodulation 1999 2:33-40.

46. Hautvast RW, DeJongste MJ, Staal MJ, van Gilst WH, Lie KI. Spinal cord stimulation in chronic intractable angina pectoris: a 
randomized, controlled efficacy study. Am Heart J 1998;136: $1114-1120$.

47. Mannheimer C, Eliasson T, Augustinsson LE, et al. Electrical stimulation versus coronary artery bypass surgery in severe angina pectoris: the ESBY study. Circulation 1998;97:1157-1163.

48. Ekre O, Eliasson T, Norrsell H, et al. Long-term effects of spinal cord stimulation and coronary artery bypass grafting on quality of life and survival in the ESBY study. Eur Heart J 2002;23:1938 1945.

49. Di Pede F, Lanza GA, Zuin G, et al.; Investigators of the Prospective Italian Registry of SCS for Angina Pectoris. Immediate and long-term clinical outcome after spinal cord stimulation for refractory stable angina pectoris. Am J Cardiol 2003;91:951-955.

50. Andersen C. Complications in spinal cord stimulation for treatment of angina pectoris: differences in unipolar and multipolar percutaneous inserted electrodes. Acta Cardiol 1997;52:325-333.

51. Bagger JP, Jensen BS, Johannsen G. Long-term outcome of spinal cord electrical stimulation in patients with refractory chest pain. Clin Cardiol 1998;21:286-288.

52. Eliasson T, Jern S, Augustinsson LE, Mannheimer C. Safety aspects of spinal cord stimulation in severe angina pectoris. Coron Artery Dis 1994;5:845-850.

53. Sanderson JE, Ibrahim B, Waterhouse D, Palmer RB. Spinal electrical stimulation for intractable angina: long-term clinical outcome and safety. Eur Heart J 1994;15:810-814.

54. Andersen C, Hole P, Oxhøj H. Will SCS treatment for angina pectoris pain conceal myocardial infraction? Abstracts of the First Meeting of the International Neuromodulation Society. Rome, 1992. Bologna: Monduzzi, 1993.

55. Andersen C, Hole P, Oxhøj H. Does pain relief with spinal cord stimulation for angina conceal myocardial infarction? Br Heart J 1994;71:419-421.

56. Hautvast R. Cardiac nociception in rats: neuronal pathways and the influence of dermal stimulation on conveyance to the central nervous system. J Mol Neurosci 2003;20:43-52.

57. Murray S, Carson KG, Ewings PD, Collins PD, James MA. Spinal cord stimulation significantly decreases the need for acute hospital admission for chest pain in patients with refractory angina pectoris. Heart 1999;82:89-92.

58. González-Darder J, González-Martínez V, Canela-Moya P. Cervical spinal cord stimulation in the treatment of severe angina pectoris. Neurosurg Q 1998;8:16-23.

59. Augustinsson LE, Eliasson T, Mannheimer C. Spinal cord stimulation in severe angina pectoris. Stereotact Funct Neurosurg 1995;65:136-141.

60. Andersen C. Does heart rate variability change in angina pectoris patients treated with spinal cord stimulation? Cardiology 1998; 89:14-18.

61. DeJongste MJL. Efficacy, safety and mechanisms of spinal cord stimulation used as an additional therapy for patients suffering from chronic refractory angina pectoris. Neuromodulation 1999; 2:188-192.

62. Hautvast RW, Brouwer J, DeJongste MJ, Lie KI. Effect of spinal cord stimulation on heart rate variability and myocardial ischemia in patients with chronic intractable angina pectoris: a prospective ambulatory electrocardiographic study. Clin Cardiol 1998;21: 33-38.

63. Meller ST, Gebhart GF. A critical review of the afferent pathways and the potential chemical mediators involved in cardiac pain. Neuroscience 1992;48:501-524.

64. Thämer V, Deussen A, Schipke JD, Tölle T, Heusch G. Pain and myocardial ischemia: the role of sympathetic activation. Basic Res Cardiol 1990;85 Suppl 1:253-266.

65. Norrsell H, Pilhall M, Eliasson T, Mannheimer C. Effects of spinal cord stimulation and coronary artery bypass grafting on myocardial ischemia and heart rate variability: further results from the ESBY study. Cardiology 2000;94:12-18.

66. Eddicks S, Maier-Hauff K, Schenk M, Müller A, Baumann G, Theres H. Thoracic spinal cord stimulation improves functional status and relieves symptoms in patients with refractory angina pectoris: the first placebo-controlled randomised study. Heart 2007;93:585-590.
67. Cook AW, Weinstein SP. Chronic dorsal column stimulation in multiple sclerosis: preliminary report. N Y State J Med 1973;73: $2868-2872$

68. Cook AW, Oygar A, Baggenstos P, Pacheco S, Kleriga E. Vascular disease of extremities. Electric stimulation of spinal cord and posterior roots. N Y State J Med 1976;76:366-368.

69. Meglio M, Cioni B, Dal Lago A, De Santis M, Pola P, Serricchio M. Pain control and improvement of peripheral blood flow following epidural spinal cord stimulation: case report. J Neurosurg 1981;54:821-823.

70. Jacobs MJ, Jörning PJ, Joshi SR, Kitslaar PJ, Slaaf DW, Reneman RS. Epidural spinal cord electrical stimulation improves microvascular blood flow in severe limb ischemia. Ann Surg 1988;207: 179-183.

71. Klomp HM, Spincemaille GH, Steyerberg EW, Habbema JD, van Urk H; ESES Study Group. Spinal-cord stimulation in critical limb ischaemia: a randomised trial. Lancet 1999;353:1040-1044.

72. Jivegård LE, Augustinsson LE, Holm J, Risberg B, Ortenwall P. Effects of spinal cord stimulation (SCS) in patients with inoperable severe lower limb ischaemia: a prospective randomised controlled study. Eur J Vasc Endovasc Surg 1995;9:421-425.

73. Broseta J, Barberá J, de Vera JA, et al. Spinal cord stimulation in peripheral arterial disease: a cooperative study. J Neurosurg 1986; 64:71-80

74. Fiume D, Palombi M, Sciassa V, Tamorri M. Spinal cord stimulation (SCS) in peripheral ischemic pain. Pacing Clin Electrophysiol 1989;12:698-704.

75. Galley D, Rettori R, Boccalon H, et al. Electric stimulation of the spinal cord in arterial diseases of the legs: a multicenter study of 244 patients [in French]. J Mal Vasc 1992;17:208-213.

76. Horsch S, Claeys L. Epidural spinal cord stimulation in the treatment of severe peripheral arterial occlusive disease. Ann Vasc Surg 1994;8:468-474.

77. Jacobs MJ, Jörning PJ, Beckers RC, et al. Foot salvage and improvement of microvascular blood flow as a result of epidural spinal cord electrical stimulation. J Vasc Surg 1990;12:354-360.

78. Sampère CT, Guasch JA, Paladino CM, Sánchez Casalongue M, Elencwajg B. Spinal cord stimulation for severely ischemic limbs. Pacing Clin Electrophysiol 1989;12:273-279.

79. Guarnera G, Furgiuele S, Camilli S. Spinal cord electric stimulation vs. femoro-distal bypass in critical ischemia of the legs. Preliminary results in a randomized prospective study [In Italian]. Minerva Cardioangiol 1994;42:223-227.

80. Ubbink DT, Vermeulen H. Spinal cord stimulation for non-reconstructable chronic critical leg ischaemia [Update of Cochrane Database Syst Rev. 2003;(3):CD004001]. Cochrane Database Syst Rev 2005;(3):CD004001.

81. Croom J, Foreman R, Chandler M, Barron K. Reevaluation of the role of the sympathetic nervous system in cutaneous vasodilation during dorsal spinal cord stimulation: are multiple mechanisms active? Neuromodulation 1998;1:91-101.

82. Linderoth B, Fedorcsak I, Meyerson BA. Is vasodilatation following dorsal column stimulation mediated by antidromic activation of small diameter afferents? Acta Neurochir Suppl (Wien) 1989;46:99-101.

83. Linderoth B, Fedorcsak I, Meyerson BA. Peripheral vasodilatation after spinal cord stimulation: animal studies of putative effector mechanisms. Neurosurgery 1991;28:187-195.

84. Linderoth B, Gunasekera L, Meyerson BA. Effects of sympathectomy on skin and muscle microcirculation during dorsal column stimulation: animal studies. Neurosurgery 1991;29:874-879.

85. Sánchez-Ledesma MJ, García-March G, Gonçalves J, et al. Role of vasoactive substances in the segmentary vasomotor response following spinal cord stimulation. An experimental study. Stereotact Funct Neurosurg 1990;54-55:224-231.

86. Hirshberg RM, Al-Chaer ED, Lawand NB, Westlund KN, Willis WD. Is there a pathway in the posterior funiculus that signals visceral pain? Pain 1996;67:291-305.

87. Ceballos A, Cabezudo L, Bovaira M, Fenollosa P, Moro B. Spinal cord stimulation: a possible therapeutic alternative for chronic mesenteric ischaemia. Pain 2000;87:99-101.

88. Krames E, Mousad DG. Spinal cord stimulation reverses pain and diarrheal episodes of irritable bowel syndrome: a case report. Neuromodulation 2004;7:82-88. 
89. Khan YN, Raza SS, Khan EA. Application of spinal cord stimulation for the treatment of abdominal visceral pain syndromes: case reports. Neuromodulation 2005;8:14-27.

90. Tiede JM, Ghazi SM, Lamer TJ, Obray JB. The use of spinal cord stimulation in refractory abdominal visceral pain: case reports and literature review. Pain Pract 2006;6:197-202.

91. Kapur S, Mutagi H, Raphael J. Spinal cord stimulation for relief of abdominal pain in two patients with familial Mediterranean fever. Br J Anaesth 2006;97:866-868.

92. Kapural L, Narouze SN, Janicki TI, Mekhail N. Spinal cord stimulation is an effective treatment for the chronic intractable visceral pelvic pain. Pain Med 2006;7:440-443.

93. Ness TJ, Gebhart GF. Visceral pain: a review of experimental studies. Pain 1990;41:167-234.

94. Mitchell GAG. Anatomy of the autonomic nervous system. Edinburgh: Livingstone, 1953.

95. Barolat G, Massaro F, He J, Zeme S, Ketcik B. Mapping of sensory responses to epidural stimulation of the intraspinal neural structures in man. J Neurosurg 1993;78:233-239.

96. North R, Kidd DH, Olin J, Sieracki JM. Spinal cord stimulation electrode design: a prospective randomized comparison of percutaneous and insulated paddle electrodes. In: Neuromodulation for pain and motor disorders. Proc. 4th Int. Congr. Int. Neuromodulation Soc., 16-20 Sept. 1998, Lucerne, Switzer- land. San Francisco: International Neuromodulation Society, 1998:211 (abstract). Available at: http://www.neuromodulation com/our-neuromodulation-publications.htm.

97. Holsheimer J, Nuttin B, King GW, Wesselink WA, Gybels JM, de Sutter P. Clinical evaluation of paresthesia steering with a new system for spinal cord stimulation. Neurosurgery 1998;42:541547; discussion 547-549.

98. Aló K, Poli P, Ghiara M, Ciaramella A, Varelli G, Yland M. The treatment of refractory reflex sympathetic dystrophy with higher frequency tripolar dual octrode spinal cord stimulation, a case report. In: Neuromodulation for pain and motor disorders. Proc. 4th Int. Congr. Int. Neuromodulation Soc., 16-20 Sept. 1998, Lucerne, Switzerland. San Francisco: International Neuromodulation Society, 1998:282 (abstract). Available at: http://www.neuromodulation.com/our-neuromodulationpublications.htm

99. Quigley DG, Arnold J, Eldridge PR, et al. Long-term outcome of spinal cord stimulation and hardware complications. Stereotact Funct Neurosurg 2003;81:50-56.

100. Spincemaille GH, Klomp HM, Steyerberg EW, van Urk H, Habbema JD; ESES Study Group. Technical data and complications of spinal cord stimulation: data from a randomized trial on critical limb ischemia. Stereotact Funct Neurosurg 2000;74:63-72. 